ISSN 1997-5902

\title{
Caractérisation physicochimique des huiles d'olive produites dans les huileries traditionnelles de la région de la Chaouia-Maroc
}

\author{
S. BOULFANE ${ }^{1 *}$, N. MAATA ${ }^{2}$, A. ANOUAR ${ }^{1}$ et S. HILALI ${ }^{3}$. \\ 'Laboratoire de Chimie Appliquée et Environnement ; Faculté des Sciences et Techniques Settat. \\ 2Laboratoire Officiel d'Analyse et de Recherche Chimique (LOARC) Casablanca \\ 3Unité de recherche Agro-ressources Marocaines et Environnement ; Faculté des Sciences et Techniques Settat. \\ ${ }^{*}$ Contact: boulfane_sa@yahoo.fr
}

Original submitted in on 21st November 2014. Published online at www.m.elewa.org on 31 st March 2015

http://dx.doi.org/10.4314/jab.v87i1.5

\section{RÉSUMÉ}

Objectif : Le but de cette étude est la détermination de la qualité des huiles d'olive issues des huileries traditionnelles de la région de la Chaouia (centre du Maroc) par une caractérisation physicochimique de leurs compositions.

Méthodologie et résultats : Des échantillons d'huiles d'olive ont été collectés à partir des huileries traditionnelles de la région. Des analyses physicochimiques concernant l'acidité libre, l'indice de peroxyde, les coefficients d'extinctions spécifiques $\mathrm{K}_{232}, \mathrm{~K}_{270}$ et $\Delta \mathrm{K}$, la teneur en chlorophylles, la teneur en composés phénoliques et la composition en acides gras ont été réalisées selon les normes du Conseil Oléicole International. Les résultats obtenus ont permis de classer les huiles étudiées en trois catégories : huile d'olive vierge, huile d'olive vierge courante et huile d'olive vierge lampante.

Conclusion et application de résultats : Les résultats obtenus confirment que les conditions de récolte, de trituration et de stockage des huiles d'olive influent la qualité de l'huile produite. Par conséquent, il faut sensibiliser les agriculteurs pour améliorer les pratiques et les techniques culturales et les propriétaires des huileries traditionnelles en ce qui concerne le stockage, la transformation et la conservation des huiles.

Mots clés: Huile d'olive, qualité, région de la Chaouia, caractérisation physicochimique.

\begin{abstract}
Physicochemical characterization of olive oils produced in traditional mills of the Chaouia area

Objective: The purpose of this study is the determination of the quality of olive oils produced in traditional mills of the Chaouia area (center of Morocco) by a physicochemical characterization of their compositions.

Methodology and Results: Samples of olive oils were collected from traditional mills of the area. Physicochemical analyzes concerning free acidity, peroxide value, specific extinctions coefficients $K_{232}$, $\mathrm{K}_{270}$ and $\Delta \mathrm{K}$, chlorophyll contents, total polyphenols content and fatty acid composition were determined according to the standards of the International Olive Oil Council . The results obtained made it possible to classify the oils studied in three categories: extra virgin olive oil, ordinary virgin olive oil and lampante virgin olive oil.

Conclusion and results application: The results obtained confirm that the conditions of harvesting, crushing and storage of olive oils affect the quality of produced oil. Consequently, it is necessary to sensitize the
\end{abstract}


farmers to improve the cultivation practices and techniques and owners of the traditional oil mills regarding the storage, the transformation and the conservation of the oils.

Keywords: Olive oil, quality, Chaouia area, physicochemical characterization.

\section{INTRODUCTION}

L'oliveraie marocaine est constituée pour plus de $96 \%$ par la variété population Picholine Marocaine (Ministère de l'Agriculture et de la Pêche Maritime, 2006). Elle se caractérise par son pouvoir d'adaptation et la qualité de ses olives qui ont une double fin: la production d'huile et le conserve d'olives. Selon la norme du Conseil Oléicole International, la qualité des huiles d'olive est un ensemble de caractéristiques physico-chimiques et organoleptiques permettant le classement des huiles en différentes catégories (Conseil Oléicole International, 2011). Cette qualité est influencée par plusieurs facteurs, tels que les techniques culturales, l'apport hydrique, la période de récolte, les techniques d'extraction et les conditions du

\section{MATERIELS ET METHODES}

Échantillonnage : L'étude a porté sur huit échantillons d'huiles d'olive ( $\left(\begin{array}{lll}E_{1} & \text { à } & E_{8}\end{array}\right)$ issus des huileries traditionnelles de la région de Chaouia durant la compagne oléicole 2012 / 2013.

Détermination de l'acidité libre: L'acidité libre, exprimée en pourcentage d'acide oléique, a été déterminée sur une prise d'essai de $1 \mathrm{~g}$ d'huile d'olive dissoute dans $50 \mathrm{ml}$ d'éthanol. Le mélange a été titré par une solution d'hydroxyde de potassium $0,1 \mathrm{~N}$ en présence de phénolphtaléine (Organisation Internationale de Normalisation ISO 660, 1996)

Détermination de l'indice de peroxyde : $1 \mathrm{~g}$ d'huile d'olive est dissoute dans $12,2 \mathrm{ml}$ du mélange d'acide acétique / chloroforme 3:2 (v / v). $15 \mathrm{ml}$ d'une solution d'iodure de potassium saturée sont additionnés au mélange. Ce dernier est placé à l'obscurité pendant 5 min. Ensuite $60 \mathrm{ml}$ d'eau distillée et $1 \mathrm{ml}$ d'une solution d'empois d'amidon sont ajoutées (une couleur violette apparaît). Le mélange obtenu a été titré par une solution de thiosulfate de sodium à $0,01 \mathrm{~N}$ (Organisation Internationale de Normalisation ISO 3960, 2007).

Analyse spectrophotométrique dans l'ultra-violet: $0,1 \mathrm{~g}$ de l'échantillon est dissout dans $10 \mathrm{ml} \mathrm{du}$ cyclohexane. Après homogénéisation, on mesure les extinctions aux longueurs d'onde $232 \mathrm{~nm}$ et $270 \mathrm{~nm}$. Pour la variation de l'extinction spécifique $(\Delta K)$, on mesure l'absorbance de l'échantillon d'huile d'olive aux stockage (Pinatel et al, 2004). Tous ces paramètres nécessitent une étude et une maitrise approfondies afin d'aboutir à une huile de bonne qualité. Dans le but d'évaluer la qualité des huiles d'olives produites dans la région de Chaouia, nous nous sommes intéressés à l'étude de la composition chimique de ces huiles. Pour ce faire, des échantillons ont été collectés à partir des huileries traditionnelles. Des analyses physicochimiques concernant l'acidité libre, l'indice de peroxyde, les coefficients d'extinctions spécifiques $\mathrm{K}_{232}, \mathrm{~K}_{270}$, la variation de l'extinction spécifique $\Delta K$, la teneur en chlorophylles, la teneur en composés phénoliques et la composition en acides gras ont été réalisées.

longueurs d'onde $266 \mathrm{~nm}$ et $274 \mathrm{~nm}$ (Conseil Oléicole International, 2011). Les valeurs d'extinctions spécifiques à $232 \mathrm{~nm}$ et $270 \mathrm{~nm}$ sont calculées selon la formule suivante :

\section{$\mathrm{K}=\mathrm{A}_{k} / \mathrm{C} \times \mathbf{S}$}

$A_{k}:$ Absorbance à la longueur d'onde $k$,

$C$ : Concentration de la solution en $\mathrm{g} / 100 \mathrm{ml}$,

$S$ : Chemin optique $(1 \mathrm{~cm})$.

Cette analyse prévoit aussi la détermination de la variation de l'extinction spécifique $\Delta K$ selon l'équation suivante : $\Delta K=K_{m}-\left(K_{m-4}+K_{m+4}\right) / 2$ où $K_{m}$ est l'extinction spécifique à la longueur d'onde d'absorbance maximale $\mathrm{m}$, aux environs de $270 \mathrm{~nm}$.

Détermination de la teneur en chlorophylles : $5 \mathrm{ml}$ d'huile d'olive sont dissout dans $5 \mathrm{ml}$ de tétrachlorure de carbone. Après homogénéisation, on mesure les absorbances à 670, 630 et $710 \mathrm{~nm}$ (Wolff, 1968). La teneur en chlorophylles est calculée selon la formule suivante :

$$
\operatorname{Chl}(\mathrm{ppm})=\frac{\text { D0670 }-\frac{(\mathrm{D} 0630+\mathrm{D} 0710)}{2}}{0,1086 \times \mathrm{L}}
$$


Détermination de la teneur en composés phénoliques: Les composés phénoliques totaux, contenus dans les échantillons étudiés, ont été extraits dans le mélange méthanol-eau $80: 20(\mathrm{v} / \mathrm{v})$. Les surnageants obtenus après centrifugation sont récupérés et le dosage a été effectué par la méthode au réactif de Folin Ciocalteu à $750 \mathrm{~nm}$, en présence d'une gamme étalon d'acide caféique (Rathjen et al, 1992).

Analyse de la composition acidique: Les esters méthyliques des acides gras sont obtenus par action du méthanol en milieu alcalin des glycérides et des acides gras libres selon la méthode standard préconisée par le Conseil Oléicole International (Conseil Oléicole International, 2001). A 0,1 g d'huile d'olive on ajoute 2 $\mathrm{ml}$ d'heptane et $0,2 \mathrm{ml}$ du $\mathrm{KOH}$ méthanolique à $2 \mathrm{~N}$. Après agitation pendant 30 secondes, la phase

\section{RESULTATS ET DISCUSSION :}

Acidité libre: L'analyse de l'acidité libre des échantillons étudiés a donné les résultats suivants. Ils sont exprimés en pourcentage d'acide oléique. On remarque que l'acidité libre des huiles d'olive de différentes zones étudiées se situe entre 1,77 et 5,83\%. Sur la base de ces résultats et selon la norme commerciale du Conseil Oléicole International, on constate qu'aucun échantillon analysé n'est de type extra vierge (acidité inferieur à 1). De même, les huiles étudiées peuvent être classées en trois catégories distinctes (Conseil Oléicole International, 2011): la classe des huiles d'olive vierge dont l'acidité libre est inferieure ou égale à 2. Cette classe renferme l'échantillon $E_{3}$. La classe des huiles d'olive vierges courantes dont l'acidité libre est inferieure ou égale à 3,3. Cette classe renferme les échantillons $E_{1}, E_{2}, E_{5}$ et $E_{6}$ et la classe des huiles d'olive vierges lampantes dont l'acidité libre est supérieure à 3,3. Cette classe supérieure héptanique ainsi obtenue est prélevée et injectée ensuite, en chromatographique en phase gazeuse. Les esters d'acides gras obtenus ont été analysés à l'aide d'un chromatographe en phase gazeuse de type HP 6890 équipé d'un détecteur à ionisation de flamme $\left(T=260^{\circ} \mathrm{C}\right)$. La colonne utilisée est une colonne capillaire de type Carbowax de dimension $30 \mathrm{~m} \times 0,32 \mathrm{~mm} \times 0,25 \mu \mathrm{m}$. Le gaz vecteur est l'azote à un débit de $2,5 \mathrm{ml} / \mathrm{min}$. Le programme de la température du four était de $140{ }^{\circ} \mathrm{C}$ à $200^{\circ} \mathrm{C}$, de 210 à $245^{\circ} \mathrm{C}$, le gradient de température est de $10^{\circ} \mathrm{C} /$ min pendant $10 \mathrm{~min}$. L'identification des pics a été réalisée en présence des témoins et le calcul des différents pourcentages d'acides gras a été fait au moyen d'un intégrateur automatique. Toutes les analyses ont été effectuées en triples et les résultats sont exprimés par les moyennes et écarts-type.

renferme les échantillons $E_{4}, E_{7}$ et $E_{8}$. Les acidités élevées que nous avons obtenus peuvent être expliquées par l'état de maturité très avancé des fruits, à l'insuffisance de précautions prises lors de la récolte ou du stockage des olives ce qui entraine la détérioration des fruits et par conséquent l'augmentation de la teneur en acides gras libres, sous l'action des lipases (El Antari et al, 2000). Cependant, les valeurs observées dans cette étude sont moins élevées que celles rapportées par Benabid et al. qui ont obtenus des valeurs entre 0,77 et $9,26 \%$ pour des huiles d'olives de différentes régions oléicoles d'Algérie (Benabid et al, 2008). Par contre, nos résultats sont très élevés par rapport à ceux rapportés par Tanouti et al. qui ont noté que l'acidité libre reste en dessous de $0,8 \%$ pour les huiles d'olive produites au Maroc oriental (Tanouti et al, 2010).

Tableau 1: Pourcentages d'acide oléique des huiles d'olive des échantillons étudiés.

\begin{tabular}{|c|c|c|c|c|c|c|c|c|}
\hline Échantillons & $E_{1}$ & $E_{2}$ & $E_{3}$ & $E_{4}$ & $E_{5}$ & $E_{6}$ & $E_{7}$ & $E_{8}$ \\
\hline $\begin{array}{l}\text { Acidité } \\
\text { oléique })\end{array} \quad \begin{array}{ll}\% & \text { acide }\end{array}$ & $\begin{array}{l}2,17 \quad \pm \\
0,01\end{array}$ & $\begin{array}{l}3,29 \\
0,01\end{array}$ & $\begin{array}{ll}1,77 & \pm \\
0,01 & \end{array}$ & $\begin{array}{ll}3,8 & \pm \\
0,01 & \end{array}$ & $\begin{array}{ll}3,1 & \pm \\
0,01 & \end{array}$ & $\begin{array}{ll}2,67 & \pm \\
0,01 & \end{array}$ & $\begin{array}{ll}5,3 & \pm \\
0,01 & \end{array}$ & $\begin{array}{l}5,83 \\
0,01\end{array}$ \\
\hline
\end{tabular}

Indice de peroxyde (IP) : Les résultats obtenus pour la teneur en peroxyde sont représentés sur le tableau 2. Les résultats sont exprimés en milliéquivalent d'oxygène actif par kilogramme d'huile (méq $\mathrm{O}_{2}$ actif / $\mathrm{kg}$ d'huile olive). 
Tableau 2 : Indice de peroxyde (IP) des huiles d'olive étudiées

\begin{tabular}{|c|c|c|c|c|c|c|c|c|}
\hline $\begin{array}{c}\text { Échantillon } \\
S\end{array}$ & $E_{1}$ & $E_{2}$ & $E_{3}$ & $E_{4}$ & $E_{5}$ & $E_{6}$ & $E_{7}$ & $E_{8}$ \\
\hline$I P$ & $\begin{array}{c}10,96 \pm 0,0 \\
1\end{array}$ & $\begin{array}{c}14,03 \pm 0,0 \\
2\end{array}$ & $\begin{array}{c}13,93 \pm 0,0 \\
2\end{array}$ & $\begin{array}{c}17,96 \pm 0,0 \\
2\end{array}$ & $\begin{array}{c}18,3 \pm 0,0 \\
2\end{array}$ & $\begin{array}{c}13,36 \pm 0,0 \\
1\end{array}$ & $\begin{array}{c}17,26 \pm 0,0 \\
1\end{array}$ & $\begin{array}{c}18,7 \pm 0,0 \\
1\end{array}$ \\
\hline
\end{tabular}

On remarque que l'indice de peroxyde (IP) oscille entre 10,96 pour l'échantillon $E_{1}$ et 18,7 méq $O_{2}$ actif / $\mathrm{kg}$ d'huile d'olive pour l'échantillon $\mathrm{E}_{8}$. Ces valeurs restent inférieures à la limite établie par la norme commerciale $\mathrm{du}$ Conseil Oléicole International pour les huiles d'olives ( $\leq 20$ méq $\mathrm{O}_{2}$ actif / $\mathrm{kg}$ d'huile olive) (Conseil Oléicole International, 2011). Cependant, les échantillons $E_{5}$ et $E_{8}$ ont des valeurs d'IP assez proches de la limite établie par le COI. Ceci peut être expliqué par une oxydation des huiles d'olive suite aux conditions de récoltes et post récoltes des olives (Ranalli, 1989). Meftah et al. ont rapporté des valeurs similaires pour des huiles d'olive originaires de la région Tadla Azilal $\left(12,07\right.$ à 18,66 méq $\mathrm{O}_{2}$ actif $/ \mathrm{kg}$ d'huile olive) (Meftah et al, 2014). Par contre Salvador et al. ont rapporté des valeurs bien plus inferieures $(7,8$ et 12,9 méq $\mathrm{O}_{2} / \mathrm{kg}$ d'huile d'olive) pour des huiles d'olive d'Espagne (Salvador et al, 2003). Pour les huiles d'olives produites au Maroc oriental, Tanouti et al. ont rapporté des teneurs en peroxydes allant de 7 à 15,4 méq $\mathrm{O}_{2} / \mathrm{kg}$ d'huile (Tanouti et al, 2010).

Absorbance dans l'ultra-violet: Les valeurs des extinctions spécifiques obtenues pour les échantillons étudiés en ultra-violet à $232 \mathrm{~nm}$ et à $270 \mathrm{~nm}$ et $\Delta \mathrm{K}$ sont présentées sur la figure ci dessous :

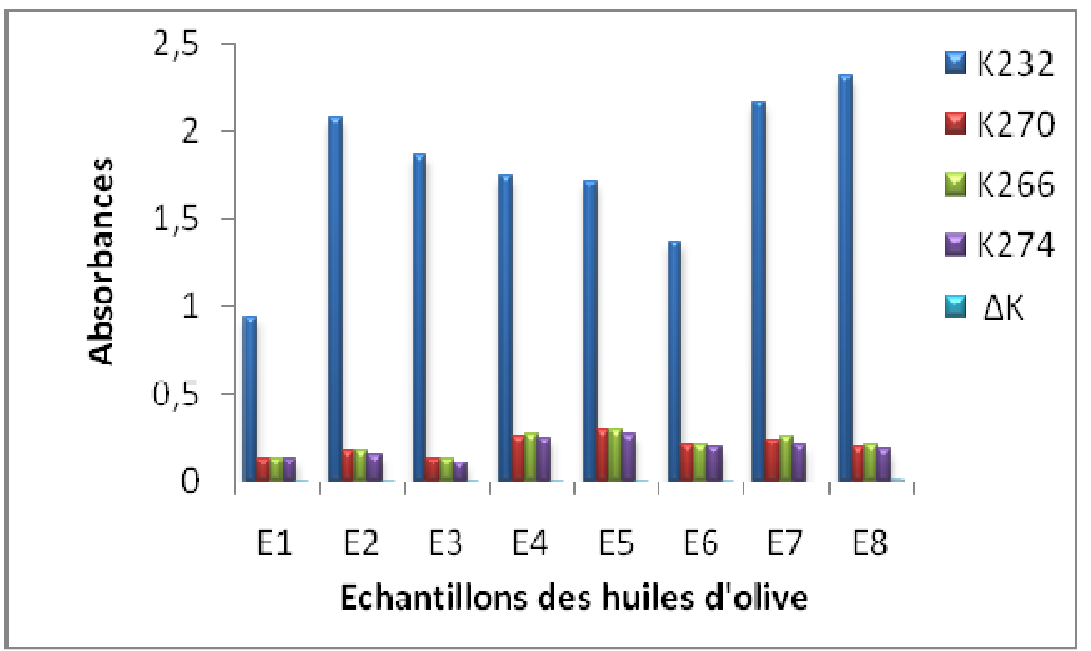

Figure 1 : Extinctions spécifiques en UV et $\Delta K$ des différents échantillons étudiés

Les valeurs des extinctions spécifiques en ultra-violet $K_{232}$ et $K_{270}$ obtenues pour les échantillons $E_{1}, E_{2}$, $E_{3}$, $E_{6}, E_{7}$ et $E_{8}$, indiquent qu'elles n'excédent pas les limites fixées par le Conseil Oléicole International pour les huiles d'olives vierges (Conseil Oléicole International, 2011). qui sont respectivement inferieures ou égales à 2,60 et 0,25 . Les échantillons $E_{4}$ et $E_{5}$ présentent des valeurs de $\mathrm{K}_{270}$ voisine de 0,3. Cette dernière représente la limite fixée par $\mathrm{COI}$ pour les huiles d'olive courantes (Conseil Oléicole International, 2011). Plusieurs facteurs peuvent expliquer ces résultats. II s'agit de la récolte tardive des olives, une exposition excessive des olives et de l'huile extraite à l'oxygène de l'air et à la lumière, voir aussi à un réchauffement de la pâte lors de la trituration (Tanouti et al, 2011). II est a noté que ces mêmes huiles, ont présenté des valeurs d'indice de peroxyde plus élevées (17,96 et 18,3 méq $\mathrm{O}_{2} / \mathrm{Kg}$ d'huile). L'extinction spécifique à $232 \mathrm{~nm}$ et à $270 \mathrm{~nm}$ d'une huile reflète son état d'oxydation. Plus son extinction à $232 \mathrm{~nm}$ est forte, plus elle est peroxydée. De même, plus l'extinction à $270 \mathrm{~nm}$ est forte, plus l'huile est riche en produits d'oxydation secondaires et traduit sa faible aptitude à la conservation (Wolff, 1968). Quant à la variation de l'extinction spécifique, elle varie d'un échantillon à l'autre mais sa valeur reste comparable à celle établie 
par le Conseil Oléicole International $(\Delta \mathrm{K}<\mathbf{0 , 0 1})$ (Conseil Oléicole International , 2011).
Teneur en pigments chlorophylliens: Les teneurs obtenues pour la chlorophylle, des échantillons étudiés, exprimée en ppm, sont représentées sur le tableau 3 :

Tableau 3 : Teneur en chlorophylles des échantillons étudiés.

\begin{tabular}{|c|c|c|c|c|c|c|c|c|}
\hline Échantillons & $E_{1}$ & $E_{2}$ & $E_{3}$ & $E_{4}$ & $E_{5}$ & $E_{6}$ & $E_{7}$ & $E_{8}$ \\
\hline Chlorophylle (ppm) & $0,9 \pm 0,01$ & $0,59 \pm 0,01$ & $0,49 \pm 0,01$ & $1,7 \pm 0,03$ & $0,48 \pm 0,02$ & $1,18 \pm 0,02$ & $1,67 \pm 0,03$ & $0,78 \pm 0,01$ \\
\hline
\end{tabular}

Les teneurs en chlorophylles, pour la plupart des échantillons étudiés, sont strictement inferieures à 2 ppm. Ces faibles teneurs sont souhaitées pour éviter l'action pro-oxydante des pigments la chlorophylliens et pour assurer ainsi une bonne conservation des huiles (Kiritsakis et al, 1987); d'où l'intérêt de produire des huiles d'olive à partir d'olives mûres et de procéder au défeuillage lors de l'extraction de l'huile. En effet, au début de la maturité des olives, la concentration en chlorophylles est élevée. Cette valeur diminue continuellement au fur et à mesure de la maturité des olives. Cette diminution est due à la dégradation de la chlorophylle en phéophytines qui confèrent à l'huile sa couleur jaune (Psomiadou et al, 2001; Ait Yacine, 2001).).

Détermination de la teneur en composés phénoliques: Les résultats des dosages des composés phénoliques sont sur le tableau 4 :

Tableau 4 : Teneur des composés phénoliques des échantillons étudiés.

\begin{tabular}{|c|c|c|c|c|c|c|c|c|}
\hline Échantillons & $E_{1}$ & $E_{2}$ & $E_{3}$ & $E_{4}$ & $E_{5}$ & $E_{6}$ & $E_{7}$ & $E_{8}$ \\
\hline $\begin{array}{c}\text { Composés } \\
\text { phénoliques } \\
(p p m)\end{array}$ & $\begin{array}{c}182,83 \pm 0, \\
01\end{array}$ & $\begin{array}{c}359,48 \pm 0, \\
02\end{array}$ & $\begin{array}{c}337,40 \pm 0, \\
01\end{array}$ & $\begin{array}{c}504,33 \pm 0, \\
03\end{array}$ & $\begin{array}{c}443,38 \pm 0, \\
01\end{array}$ & $\begin{array}{c}294,12 \pm 0, \\
01\end{array}$ & $\begin{array}{c}514,93 \pm 0, \\
01\end{array}$ & $\begin{array}{c}446,03 \pm 0, \\
01\end{array}$ \\
\hline
\end{tabular}

Les résultats obtenus montrent que les huiles d'olive étudiées renferment une quantité appréciable de composés phénoliques. Cette quantité oscille entre 182,83 et 514,93 ppm. Les résultats que nous avons obtenus sont en accord avec ceux rapportés par AbuReidah et al. qui ont trouvé des valeurs allant de 318.99 à $469.96 \mathrm{ppm}$ pour des huiles palestiniennes (M. Abu-Reidah et al, 2013).Cependant, elles sont bien élevées que celles obtenues pour les huiles d'olives de la région de Tadla Azilal (179,9 à $281,35 \mathrm{mg} / \mathrm{kg})$ (Meftah et al, 2014). Les composés phénoliques passent dans l'huile lors de son extraction. Ils sont considérés comme des antioxydants naturels qui protègent l'huile contre l'oxydation et lui confèrent une meilleure stabilité lors du stockage et une saveur amère (Tanouti et al, 2011). Les variations des teneurs observées peuvent être dues à la différence du degré de maturité des olives avant trituration, (récolte précoce des olives) mais dépendent également de la variété cultivée et de la zone géographique (Garcia et al, 2003). En effet, les huiles d'oliveraie située en altitude sont plus riches en phénols que les oliveraies des plaines (Ocakoglu, 2008). La présence des feuilles lors de broyage des olives peut aussi augmenter la concentration en composés phénoliques dans les huiles d'olive (Boudhioua et al, 2008).

Détermination de la composition en acide gras : Les résultats obtenus de la chromatographie en phase gazeuse sont présentés dans le tableau ci-dessous :

Les résultats obtenus pour les 8 échantillons étudiés montrent que la composition en acides gras des huiles d'olive analysées répond aux normes fixées par le Conseil Oléicole International (Conseil Oléicole International, 2009). Cette composition acidique est variable. En effet, les pourcentages de l'acide oléique (C18:1) varient entre $67,23 \%$ pour l'échantillon $E_{4}$ et $73,2 \%$ pour l'échantillon $E_{1}$ alors que les pourcentages de l'acide linoléique (C18:2) varient entre 11,8\% pour l'échantillon $E_{1}$ et $17,14 \%$ pour l'échantillon $E_{4}$. Ces deux acides gras sont donc les prédominants, suivi des acides palmitique ( $\mathrm{C} 16: 0)$ et stéarique (C18:0) à des taux de l'ordre de 10,09\% pour l'échantillon $\mathrm{E}_{3}$ à 10,54 $\%$ pour l'échantillon $\mathrm{E}_{2}$ et de 2,16\% pour l'échantillon $E_{1}$ à 2,62 \% pour l'échantillon $E_{5}$ respectivement. Les acides gras mineurs dont les pourcentages obtenus pour les échantillons étudiés ne dépassent guère $4 \%$, 
Boulfane et al. J. Appl. Biosci. Caractérisation physicochimique des huiles d'olive produites dans les huileries traditionnelles de la région de la Chaouia-Maroc

sont formés par l'acide palmitoléique, stéarique, linoléique, arachidique et gadoléique alors que les acides gras présents à l'état de traces dont les pourcentages sont inférieurs à 0,2 \% sont représentés par, l'acide margaroléique.

Tableau 5 : Pourcentages des acides gras des échantillons étudiés.

\begin{tabular}{|c|c|c|c|c|c|c|c|c|c|}
\hline & $\mathrm{E}_{1}$ & $\mathrm{E}_{2}$ & $\mathrm{E}_{3}$ & $\mathrm{E}_{4}$ & $\mathrm{E}_{5}$ & $\mathrm{E}_{6}$ & $\mathrm{E}_{7}$ & $\mathrm{E}_{8}$ & $\begin{array}{c}\text { Normes } \\
\text { COI }\end{array}$ \\
\hline Palmitique C16:0 & 10,43 & 10,54 & 10,09 & 10,49 & 10,26 & 10,32 & 10,13 & 10,46 & $7,5-20,0$ \\
\hline $\begin{array}{c}\text { Palmitoléique } \\
\text { C16:1 }\end{array}$ & 0,83 & 0,7 & 0,7 & 0,7 & 0,71 & 0,67 & 0,77 & 0,79 & $0,3-3,5$ \\
\hline $\begin{array}{c}\text { Margarique C17:0 } \\
\text { Margaroléique } \\
\text { C17:1 }\end{array}$ & 0,06 & 0,06 & 0,02 & 0,13 & 0,03 & 0,11 & 0,04 & - & $<0,3$ \\
\hline Stéarique C18:0 & 2,16 & 2,42 & 2,46 & 2,42 & 2,62 & 2,16 & 2,43 & 2,17 & $0,5-5,0$ \\
\hline Oléique C18:1 & 73,2 & 69,38 & 70,02 & 67,23 & 69,53 & 72,19 & 71,3 & 71,74 & $55,0-$ \\
\hline Linoléique C18:2 & 11,8 & 15,22 & 14,93 & 17,14 & 15,08 & 12,87 & 13,57 & 13,25 & $3,5-21,0$ \\
\hline Linolénique C18:3 & 0,89 & 1,02 & 1,05 & 1,25 & 1,06 & 0,94 & 1,03 & 0,97 & $0,0-1,5$ \\
\hline Arachidique C20:0 & 0,28 & 0,27 & 0,3 & 0,32 & 0,35 & 0,3 & 0,36 & 0,29 & $<0,6$ \\
\hline Gadoléique C20:1 & 0,26 & 0,27 & 0,31 & 0,2 & 0,3 & 0,29 & 0,31 & 0,33 & $<0,4$ \\
\hline AGS & 12,93 & 13,29 & 12,87 & 13,36 & 13,26 & 12,89 & 12,96 & 12,92 & \\
\hline AGI & 87,07 & 86,69 & 87,11 & 86,63 & 86,74 & 87,02 & 87,04 & 87,08 & \\
\hline AGI/AGS & 6,73 & 6,52 & 6,77 & 6,48 & 6,54 & 6,75 & 6,72 & 6,74 & \\
\hline
\end{tabular}

La présence de l'acide gras polyinsaturé : acide linoléique (C18:2) avec un pourcentage important comparativement aux autres acides gras insaturés peut être expliquée par la présence d'une enzyme, l'Oléate desaturase qui transforme l'acide oléique (C18:1) en acide linoléique (C18:2) au cours de la maturation du fruit (Gutierrez et al, 1999). Les pourcentages en acide oléique dans les huiles d'olive étudiés sont similaires aux valeurs trouvées par Abu-Reidah et al. qui ont trouvé des valeurs allant de 67.24 à $72.27 \%$ pour des huiles palestiniennes (M. Abu-Reidah et al, 2013). Cependant, ils sont un peu élevés que les valeurs rapportées par Issaoui et al pour les huiles tunisiennes (54.6 à $66.8 \%$ ) (Issaoui et al, 2010). II faut également signaler que la composition en acides gras obtenue

\section{CONCLUSION}

L'étude des paramètres de qualité des huiles d'olives issues de la région de Chaouia a permis de classer les huiles d'olive étudiées en trois catégories: huiles d'olive vierges, vierges courantes et vierges lampantes. On note l'absence des huiles d'olive de qualité extra vierge dans les échantillons étudiés. Les facteurs responsables d'acidité élevée sont liés au non révèle une prédominance des acides gras mono insaturés. Le pourcentage des acides gras insaturés $(A G I)$ varie légèrement, en fonction des échantillons étudiés. II varie entre 86,63 pour $E_{4}$ et $87,11 \%$ pour $E_{3}$. De même le pourcentage des acides gras saturés est variable, il oscille entre 12,89 pour $E_{3}$ et $13,49 \%$ pour $E_{4}$. Le rapport entre les acides gras insaturés sur les acides gras saturés (AGI/AGS) montre également une fluctuation en fonction des échantillons étudiés. $\mathrm{Ce}$ rapport oscille entre 6,48 pour $E_{4}$ et 6,77 pour $E_{3}$. Ce rapport est plus élevé ce qui confère à l'huile d'olive une plus grande stabilité à l'auto-oxydation et une valeur nutritionnelle importante (Ryan et al, 1998; Abaza et al, 2003).

respect des bonnes pratiques de récolte, de trituration et de stockage des huiles d'olives. La composition en acides gras a révélé que les huiles d'olive étudiées sont très riche en acides gras insaturés: C18:1, C18:2 et C18:3. En effet, le rapport des acides gras insaturés sur les acides gras saturés est élevé et oscille entre 6,42 et 6,76 .d'où leurs intérêts dans la conservation des 
huiles d'olive et dans la nutrition humaine. Pour valoriser d'avantage ces huiles régionales et leur donner une valeur ajoutée, il est nécessaire de sensibiliser les agriculteurs pour améliorer les pratiques

\section{RÉFÉRENCES BIBLIOGRAPHIQUES :}

Ministère de l'Agriculture et de la Pêche Maritime, (2006). Données générales sur l'agriculture marocaine. Les cultures arboricoles. Direction de la Statistique.

Conseil Oléicole International (2011). Norme commerciale applicable aux huiles d'olive et aux l'huiles de grignions d'olive. T. 15/NC $n^{\circ}$ 3/Rév. 6.

Pinatel C., Petit C., Ollivier D et Artaud J. (2004). Outil pour l'amélioration organoleptique des huiles d'olive vierges. Oléagineux, Corps Gras, Lipides .11(3) : 217-222.

Organisation Internationale de Normalisation: ISO 660 : (1996) .Corps gras d'origines animale et végétale -Détermination de l'indice d'acide et de l'acidité.

Organisation Internationale de Normalisation: ISO 3960 : (2007). Corps gras d'origines animale et végétale - Détermination de l'indice de peroxyde - Détermination avec point d'arrêt iodométrique.

Wolff J-P. (1968). Manuel d'analyse des corps gras. Edition. Azoulay, Paris.

Rathjen, A. H. et Robinson, S. P. (1992). Characterization of a variegated grapevine mutant showing reduced polyphénol oxidase activity. Aust. J. Plant Physiol. 19 : 43-54.

Conseil Oléicole International (2001). Préparation des esters méthyliques d'acides gras de l'huile d'olive et de l'huile de grignons d'olive. T.20/Doc. N $\mathrm{N}^{0} 24$.

El Antari, A., Hilal, A., Boulouha, B. et El Moudni, A. (2000). Influence of the variety, environment and cultural techniques on the characteristics of olive fruits and the chemical composition of extra virgin olive oil in Morocco. Olivae. (80): 29-36.

Benabid H., Naamoune H., Noçairi H. et Rutledge D. (2008). Application of chemometric tools to compare Algerian olive oils produced in different locations. Journal of Food, Agriculture \& Environment. 6 (2) : 43-51.

Tanouti K., Elamrani A., Serghini Caid H., Khalid A., Bahetta Y., Benali A., Harkous M.et Khiar M. (2010). Caractérisation d'huiles d'olive et les techniques culturales et les propriétaires des huileries traditionnelles en ce qui concerne le stockage, la transformation et la conservation des huiles.

produites dans des coopératives pilotes (Lakrarmaet Kenine) au niveau du Maroc oriontal. Les technologies de Laboratoire, $5: 18-26$.

Ranalli A. (1989). Aspects et problèmes se rattachant au stockage et à la conservation de l'huile d'olive. Olivae. $272: 11-18$.

Meftah H., Latrache H., Hamadi F., Hanine H., Zahir H. et El louali M. (2014). Comparaison des caractéristiques physicochimiques des huiles d'olives issues de différentes zones de la région Tadla Azilal (Maroc). J. Mater. Environ. Sci. 5 (2) : 641-646

Salvador M. D., Aranda F., Gomez-Alonso S. et Fregapane G. (2003). Influence of extraction system, production year and area on Cornicabra virgin olive oil : a study of five crop seasons. Food Chemistry. $80: 359-366$.

Tanouti K., Serghini-Caid H., Chaieb E., Benali A., Harkous M.et Elamrani A. (2011). Quality improvement of olive oils produced in the eastern Morocco. Les technologies de Laboratoire. 6 (22) : 1-12.

Kiritsakis, A. et Markakis, P. (1987). Olive oil: a review. Advance Food Research. 31: 118125.

Psomiadou, E.et Tsimidou, M., (2001). Pigments in Greek virgin olive oils: occurrence and levels. Journal of the Science of Food and Agriculture. 81: 640-647.

Ait Yacine Z. (2001). Etude des facteurs déterminant la meilleure période de récolte des

Olives (var. Picholine marocaine) destinées à la trituration dans le Tadla. Thèse de Doctorat d'état ès-Sciences, Université Mohamed ler, Faculté des Sciences, Oujda.

M. Abu-Reidah I., Yasin M., Urbani S., Servili M. et Montedoro G. (2013). Study and characterization of Palestinian monovarietal Nabali virgin olive oils from northern West Bank of Palestine. Journal Food Research International. 54 (2) : 1959-1964.

Garcia A., Brenes M., Garcia P., Romero C. et Garrido A. (2003). Phenolic content of commercial 
olive oils. European Food Research and Technology. $216(6): 520-525$.

Ocakoglu D. (2008). Classification of Turkish virgin olive oils based on their phenolic profils. Thesis, Master of Science in food engineering and science - Izmir institute of technology Turkish.

Boudhioua N., Ben Slimen I., Bahloul N. et Kechaou N. (2008). Etude du séchage par infrarouge de feuilles d'olivier d'origine tunisienne. Revue des Energies Renouvelables SMSTS'Alger: $111-116$.

Conseil Oléicole International. (2009). Norme commercial applicable aux huiles d'olive et aux huiles de grignon d'olive. $\mathrm{COI} / \mathrm{NCn}^{\circ} 3$ / Rev.4.

Gutierrez F., Jimenez B., Ruiz A. et albi M.A. (1999). Effect of olive ripeness on the oxidative stability of virgin olive oil extracted from the varieties Picual and Hojiblanca and on the different components involved. J Agric. Food Chemistry. $47:$ 121-127.

Issaoui M., Flamini G., Brahmi F., Dabbou S, Ben Hassine K., Taamali A., Chehab H., Ellouz M, Zarrouk M. et Hammamia M. (2010). Effect of the growing area conditions on differentiation between Chemlali and Chétoui olive oils. Food Chemistry. 119 : 220-225.

Ryan D., Robards K. et Lavee S. (1998). Evolution de la quantité de l'huile d'olive. Olivae. 72 : 23-41.

Abaza L., Ben Temime S., M'Sallem M., Daoud D., Zarrouk M. et Cherif A. (2003). Etude comparative de la lipogenèse chez quelques variétés d'oliviers cultivées en Tunisie. Riv. Ital. Dell Sost. Gr. 80: 297-306. 